\title{
Incidence of coagulase-negative staphylococcal bacteremia among ICU patients: decontamination studies as a natural experiment
}

\author{
James C Hurley ${ }^{1,2}$ iD \\ Received: 22 August 2019 / Accepted: 4 November 2019 / Published online: 4 December 2019 \\ (C) Springer-Verlag GmbH Germany, part of Springer Nature 2019
}

\begin{abstract}
The epidemiology of coagulase-negative staphylococcal (CNS) bacteremia among adult ICU patients remains unclear. Decontamination studies among ICU patients provide a unique opportunity to study the impacts of different diagnostic criteria, exposure to various decontamination interventions, and various other factors, on its incidence over three decades. Decontamination studies among ICU patients reporting CNS bacteremia incidence data were obtained mostly from recent systematic reviews. The CNS bacteremia incidence within component (control and intervention) groups of decontamination studies was benchmarked versus studies without intervention (observational groups). The impacts of antibiotic versus chlorhexidine decontamination interventions, control group concurrency, publication year, and diagnostic criteria were examined in meta-regression models. Among non-intervention (observational) studies which did versus did not specify stringent ( $\geq 2$ positive blood cultures) diagnostic criteria, the mean CNS bacteremia incidence per 100 patients (and 95\% CI; $n$ ) is $1.3(0.9-2.0 ; n=23$ ) versus $3.6(1.8-6.9 ; n=8)$, respectively, giving an overall benchmark of $1.8(1.2-2.4 ; n=31)$. Versus the benchmark incidence, the mean incidence is high among concurrent control (5.7; 3.6$9.1 \%)$ and intervention $(5.2 ; 3.6-6.9 \%)$, but not non-concurrent control (1.0; 0.4-3.9\%) groups of 21 antibiotic studies, nor among eleven component groups of chlorhexidine studies. This high incidence remained apparent $(p<0.01)$ in meta-regression models adjusting for group wide factors such as diagnostic criteria and publication year. The incidence of CNS bacteremia within both intervention and concurrent (but not non-concurrent) control groups of antibiotic-based decontamination studies are unusually high even accounting for variable diagnostic criteria and other factors.
\end{abstract}

Keywords Coagulase-negative staphylococci $\cdot$ Bacteremia $\cdot$ Intensive care unit $\cdot$ Decontamination

\section{Introduction}

Coagulase-negative staphylococci (CNS) bacteremias are common among adult ICU patients. However, whether CNS bacteremia is more common in recent years, and whether antibiotic use is a risk factor, remains unclear

Electronic supplementary material The online version of this article (https://doi.org/10.1007/s10096-019-03763-0) contains supplementary material, which is available to authorized users.

James C Hurley

jamesh@bhs.org.au; hurleyjc@unimelb.edu.au

1 Melbourne Medical School, University of Melbourne, Melbourne, Australia

2 Division of Internal Medicine, Internal Medicine Service, Ballarat Health Services, PO Box 577, Ballarat 3353, Australia
[1-3]. In addition, its overall clinical importance is often unclear $[4,5]$ and diagnostic criteria requiring more than one positive blood culture are in common use [6-9].

Moreover, the impact of decontamination interventions, such as topical antibiotic prophylaxis (TAP), being used either alone (selective oropharyngeal decontamination; SOD) or combined (selective digestive decontamination; SDD) with protocolized parenteral antibiotic prophylaxis (PPAP) within SDD/SOD regimens on CNS bacteremia remains unclear as CNS are generally not subject to surveillance $[10,11]$. The TAP components generally target Gram-negative bacteria and are non-absorbable. Additionally, chlorhexidine-based decontamination strategies have emerged for consideration although the impact of these on overall bacteremia incidence is less apparent than for SDD/SOD interventions [12-15].

The objectives here are threefold: to develop a benchmark for CNS bacteremia among published observational studies of ICU 
patients; to survey and compare the incidence of CNS bacteremia within the component (control and intervention) groups decanted from decontamination studies, versus these external benchmarks; and to model by meta-regression its association with various group level factors among the component groups within these studies.

\section{Materials and methods}

Being an analysis of published work, ethics committee review of this study was not required.

\section{Study selection and decant of groups}

The literature search and study decant used here is outlined in Fig. 1 and are as described previously [16,
17]. Most studies were obtained from systematic reviews of decontamination interventions in ICU patients as listed previously. These were screened against the following eligibility criteria. Inclusion criteria are as follows: incidence data for CNS bacteremia was extractable as an incidence proportion. Exclusion criteria are as follows: studies limited to patients with the acute respiratory distress syndrome and studies of topical antibiotics used in the context of an ICU outbreak. A hand search was undertaken for additional studies and additional sources of data not identified within systematic reviews.

The studies were streamed into one of three categories: studies in which there was no intervention (observational studies), studies with antibiotic prophylaxis in any formulation, and studies of chlorhexidine-based interventions.
Fig. 1 Search method, screening criteria and resulting classification of eligible studies and subsequent decant of component groups. The six numbered arrows are as follows: (1) An electronic search for systematic reviews containing potentially eligible studies using search terms; "ventilator associated pneumonia," "mechanical ventilation," "intensive care unit," each combined with either "metaanalysis" or "systematic review" up to December 2017, (2) studies were streamed into one of three categories; studies in which there was no intervention (observational studies), and studies of either chlorhexidine-based or antibioticbased interventions; (3) all studies were reviewed for potentially eligible studies and screened against inclusion and exclusion criteria;

(4) a hand search was undertaken for additional studies; (5) eligible studies were then collated and any duplicate studies were removed; (6) the component groups were decanted from each study being control (rectangles), intervention (ovals), and observation (diamond) groups. Note: the total numbers do not tally as some systematic reviews provided studies in more than one category and some studies provided groups in more than one category

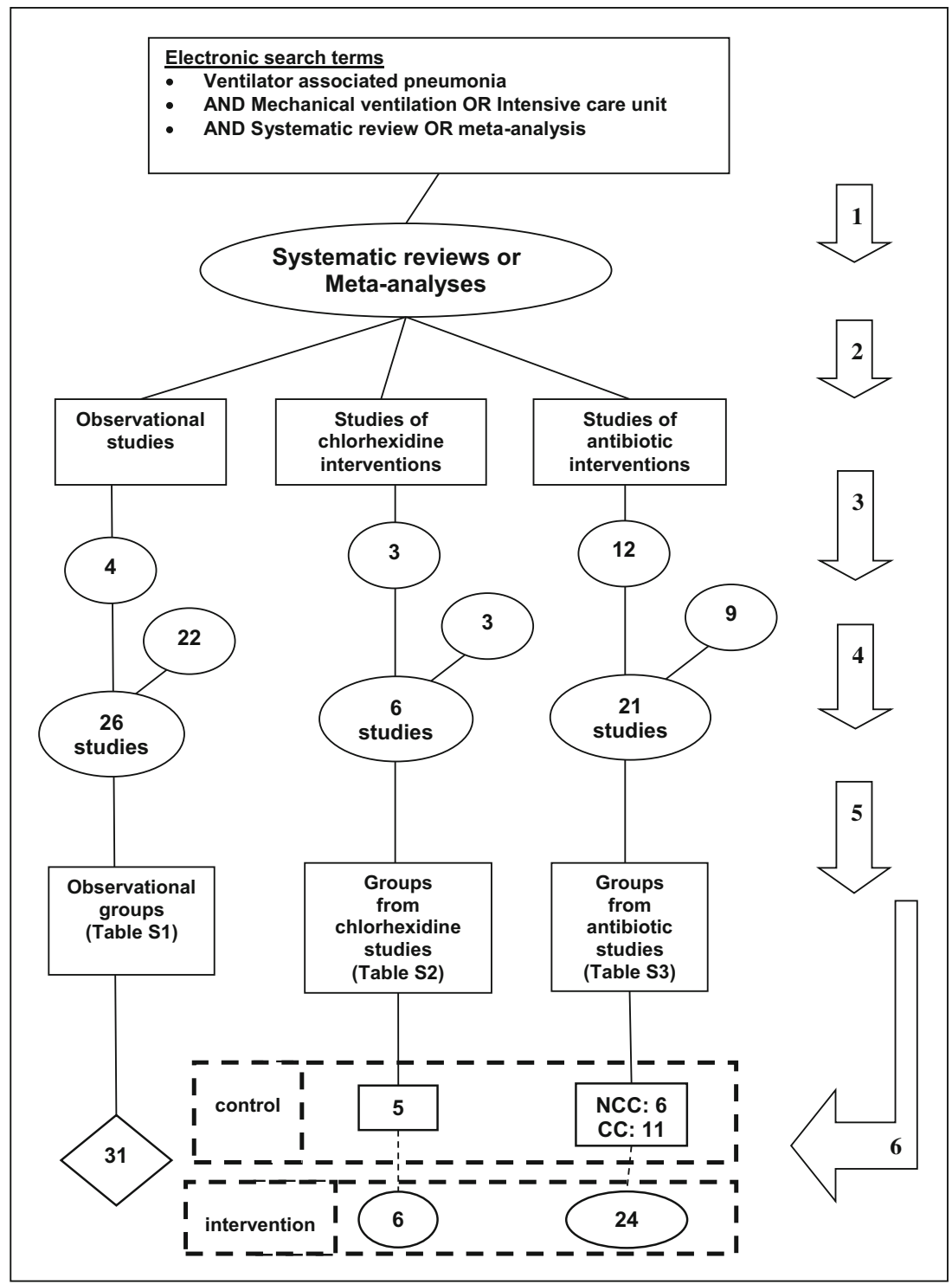




\section{Outcomes of interest}

The overall and CNS bacteremia incidences were expressed as a proportion using the number of patients with prolonged (> $24 \mathrm{~h}$ ) stay in the ICU as the denominator. In addition, the following were also extracted: the proportion of each group receiving mechanical ventilation (MV), the proportion of admissions for trauma, the year of study publication, and whether the study ICU was located in North America (Canada or the USA). The use of stringent versus less stringent diagnostic criteria for CNS bacteremia was examined. Studies which stated that either CDC criteria [9] were followed or that $\geq 2$ positive blood cultures was a specific requirement for CNS bacteremia were accepted as meeting stringent criteria.

\section{Effect sizes}

The study-specific and overall summary effect sizes and associated $95 \%$ confidence interval for each of the antibiotic-based and chlorhexidine-based decontamination interventions against bacteremia overall were calculated using the Der Simonian-Laird random-effect methods of meta-analysis using the "metan" command in Stata 14.2 (StataCorp., College Station, TX, USA).

\section{Benchmarking}

Caterpillar plots of the overall and CNS bacteremia incidence were generated to facilitate a visual survey. These were generated from the incidence data as follows. Bacteremia incidence data for each group were logit transformed to generate caterpillar plots using the "metan" command previously [16, 17]. For CNS bacteremia, this transformation proceeds as follows: with the number of patients as the denominator $(D)$, the number of patients with CNS bacteremia as the numerator $(N)$, and $R$ being the CNS bacteremia proportion $(N / D)$, the logit (CNS bacteremia) is $\log (N /(D-N))$ and its variance is $1 /(D \times$ $R \times(1-R))$. Note that for any group with a zero event rate $(N=$ 0 ), the addition of the continuity correction (i.e., $N+0.5$ ) is required to avoid indeterminate transformations of the logit proportion and its variance. For each bacteremia type, the benchmark is the summary incidence as derived in the caterpillar plot of the observational groups. This benchmark is used as a reference line in the plot of the component groups from the antibiotic and chlorhexidine-based studies.

\section{Meta-regression}

Meta-regression models for each of overall and CNS bacteremia incidence data was undertaken using random effects methods using the calculated logits and logit variances with the metareg command. Each meta-regression model incorporates group level factors as predictors being the type of study, observational or intervention, type of intervention, being chlorhexidine- or antibiotic-based decontamination, and type of component group being membership of an observational group, a control group, or an intervention group. For the antibiotic-based decontamination studies, whether the control groups were concurrent versus non-concurrent to the intervention groups in the study were additional predictor variables. All predictor variables were entered into each model without any preselection step. The meta-regression was repeated with the following tests of sensitivity: firstly, using the method of generalized estimating equations; secondly, including studies which had been excluded because the mean length of ICU stay was less than 4 days.

\section{Results}

\section{Characteristics of the studies}

Of the 52 studies identified by the search (Fig. 1), most were sourced from previous searches (Table $1[16,17]$ ), most were published between 1985 and 2015, and a minority originated from either North American or trauma ICU's (Tables S1-S3, available as Supplementary data). The majority of decontamination studies originated from five systematic reviews as listed in the ESM.

From 52 studies, 83 component groups were decanted with 31 groups from observational studies (Table S1), and 52 groups from studies of either chlorhexidine (Table S2) or antibiotic-based (Table S3) decontamination regimens. Eight studies had either no control group or more than one control, intervention, or observational group. Most groups from observational studies had more than 600 patients per group versus less than 120 patients in the majority of all remaining groups. The majority of groups across all categories included patients receiving prolonged mechanical ventilation.

Among the studies of antibiotic-based decontamination, 19 of the 26 TAP regimens contained topical polymyxin. Among the chlorhexidine decontamination methods, oropharyngeal applications were used in four and body washings were used in two studies.

The use of stringent diagnostic criteria for CNS bacteremia ( $\geq 2$ positive blood cultures) was reported among all the studies from North America $(n=15)$ versus most studies from the rest of the world (25 of 46) and was similar over the thirty year period. Any increase in CNS incidence over the period was non-significant as a linear trend (Fig. S7).

\section{Bacteremia overall}

The level of protection apparent against overall bacteremia among the various decontamination interventions 
Table 1 Characteristics of studies

\begin{tabular}{|c|c|c|c|c|}
\hline \multirow[t]{2}{*}{ Variable } & \multirow[t]{2}{*}{ Observational (no intervention) } & \multirow[t]{2}{*}{ Chlorhexidine studies } & \multicolumn{2}{|l|}{ Antibiotic studies } \\
\hline & & & Non-concurrent control & Concurrent control \\
\hline Supplemental material & Table S1 & Table S2 & Table S3 & Table S3 \\
\hline Number of studies ${ }^{\text {a }}$ & 26 & 6 & 6 & 24 \\
\hline Origin from systematic review & 4 & 3 & 1 & 11 \\
\hline Publication year (range) & 1985-2014 & $2000-2016$ & 1984-2018 & $1984-2018$ \\
\hline LOS $<5$ days $(n)^{\mathrm{b}}$ & 2 & 0 & 1 & 1 \\
\hline MV for $>48 \mathrm{~h}$ for $<90 \%^{\mathrm{c}}$ & 10 & 1 & 2 & 3 \\
\hline Trauma ICUs $(n)^{\mathrm{d}}$ & 3 & 0 & 2 & 4 \\
\hline Liver transplant $\mathrm{ICU}^{\mathrm{e}}$ & 0 & & 0 & 6 \\
\hline Stringent criteria ${ }^{\mathrm{g}}$ & 19 & 4 & 2 & 7 \\
\hline North American ICUs $(n)^{\mathrm{e}}$ & 11 & 2 & 0 & 3 \\
\hline $\begin{array}{l}\text { Patients per study group; } \\
\text { median (IQR) } \mathrm{f}\end{array}$ & $680 ; 206-2640$ & $114 ; 30-164$ & $99 ; 59-925$ & $90 ; 41-200$ \\
\hline $\begin{array}{l}\text { Bacteremia prevention effect size }{ }^{\mathrm{h}} \\
\quad(\text { summary odds ratio; } 95 \% \text { CI, } n)\end{array}$ & & $\begin{array}{l}0.89 ; 0.74-1.05 \\
\quad \text { (6); Fig. S1 }\end{array}$ & $\begin{array}{l}0.78 ; 0.6-0.91 \\
\text { (7); Fig. S1 }\end{array}$ & $\begin{array}{l}0.85 ; 0.66-1.09 \\
\text { (11); Fig. S1 }\end{array}$ \\
\hline \multicolumn{5}{|l|}{$\begin{array}{l}\text { Overall bacteremia incidence } \\
\text { per } 100 \text { patients }\end{array}$} \\
\hline $\begin{array}{l}\text { - Observational or control } \\
\text { (mean; } 95 \% \mathrm{CI} ; n)\end{array}$ & $8.7 ; 6.9-10.0(31)$ & $5.2 ; 2.4-10.9(5)$ & $10.0 ; 4.7-19.8(6)$ & $19.8 ; 14.2-26.9(10)$ \\
\hline - Intervention (mean; $95 \% \mathrm{CI} ; n$ ) & & $6.3 ; 5.2-8.3(6)$ & $10.9 ; 7.6-15.4(23)$ & \\
\hline \multicolumn{5}{|l|}{$\begin{array}{l}\text { Overall } S \text {. aureus bacteremia } \\
\text { incidence per } 100 \text { patients }\end{array}$} \\
\hline $\begin{array}{l}\text { - Observational or control } \\
\text { (mean; } 95 \% \mathrm{CI} ; n)\end{array}$ & $1.5 ; 1.1-2.0(29)$ & $2.7 ; 1.2-5.7(5)$ & $1.6 ; 0.4-5.7(6)$ & $4.3 ; 2.7-6.9(10)$ \\
\hline - Intervention (mean; 95\% CI; $n$ ) & & $1.3 ; 1.1-1.8(6)$ & $2.4 ; 1.3-4.7(23)$ & \\
\hline \multicolumn{5}{|l|}{$\begin{array}{l}\text { CNS bacteremia incidence per } \\
100 \text { patients }\end{array}$} \\
\hline $\begin{array}{l}\text { - Observational or control } \\
\text { (mean; } 95 \% \text { CI; } n)\end{array}$ & $1.8 ; 1.2-2.4^{\mathrm{j}, \mathrm{k}}(31)$ & $2.4 ; 1.6-3.2(5)$ & $1.0 ; 0.4-3.9(6)$ & $5.7 ; 3.6-9.1(11)$ \\
\hline - Intervention (mean; $95 \% \mathrm{CI} ; n)$ & & $2.2 ; 0.7-5.7(6)$ & $5.2 ; 3.6-6.9(24)$ & \\
\hline
\end{tabular}

${ }^{a}$ Note: Several studies had one, $\geq 1$, or no control and or one or $\geq 1$ intervention group. Hence, the number of groups does not equal the number of studies

${ }^{\mathrm{b}} \mathrm{LOS}<5$ days $=$ mean length of stay for the group of less than 5 days

${ }^{\mathrm{c}} \mathrm{MV}$ for $>48 \mathrm{~h}$ for $<90 \%$ = studies for which less than $90 \%$ of patients were reported to receive $>48 \mathrm{~h}$ of MV

${ }^{\mathrm{d}}$ Trauma ICU arbitrarily defined as an ICU with more than $50 \%$ of admissions for trauma

${ }^{\mathrm{e}} \mathrm{PPAP}=$ protocolized parenteral antibiotic prophylaxis

${ }^{\mathrm{f}}$ Study originating from an ICU in the USA or Canada

${ }^{g}$ Groups for which stringent criteria were applied for CNS bacteremia (e.g., an explicit statement that at least 2 positive blood cultures were required)

${ }^{\mathrm{h}}$ This is the effect of overall bacteremia prevention

${ }^{i}$ Data is median and inter-quartile range (IQR) for numbers in the observation or control groups

${ }^{\mathrm{j}}$ This is the overall CNS bacteremia benchmark as derived in Fig. S1

${ }^{\mathrm{k}}$ For groups using $\geq 2$ positive blood culture as diagnostic criteria, the mean incidence is $1.3 ; 0.9-2.0$ (23) versus 3.6 ; 1.8-6.9 (8) for those not using this criteria (Fig. S5)

included here was statistically marginal (Table 1; Fig. S1). The mean incidence of overall bacteremia (Fig. 2, Fig S2S3), and S. aureus bacteremia (Fig S4), was in each case higher among the $\mathrm{CC}$ groups of the studies of antibioticbased decontamination methods versus the respective mean bacteremia incidence among the observational groups (Table 1).

\section{CNS bacteremia}

The CNS bacteremia benchmark here is 1.8 per 100 patients (Fig. S5). The mean incidence of CNS bacteremia was higher among both the $\mathrm{CC}$ groups and the intervention groups of the studies of antibiotic-based decontamination methods versus the benchmark whereas the mean incidence derived from the 
other component group categories was each similar to this benchmark (Table 1, Fig. 3, Fig. S7).

Among non-intervention (observational) studies which did versus did not specify stringent diagnostic criteria, the mean CNS bacteremia incidence per 100 patients (and 95\% CI) is $1.3(0.9-2.0 ; n=23)$ versus $3.6(1.8-6.9 ; n=8)$, respectively.

Seven studies were excluded because the mean length of stay was less than 4 days (Table S4). Among these studies, the CNS bacteremia incidence was $<1 \%$ in eight groups of 2 multi-ICU studies of chlorhexidine decontamination methods (131,986 patients) and $>1 \%$ in seven of eight groups of five studies of antibiotic-based decontamination regimens (577 patients) (Table S4).

In a meta-regression model adjusting for all the group level factors as detailed in Table 2, memberships of either a CC control $(p=0.003)$ or an intervention $(p=0.002)$ group within a study of antibiotic-based decontamination methods were significantly associated with higher CNS bacteremia incidence. The magnitude of this association was similar to that of the significant association with the application of loose versus stringent diagnostic criteria for CNS bacteremia. By contrast, membership of an NCC control group $(p=0.37)$ of an antibiotic-based decontamination study or membership of a control or intervention group of a chlorhexidine-based decontamination study $(p=\mathrm{NS})$ was not associated with CNS bacteremia incidence.

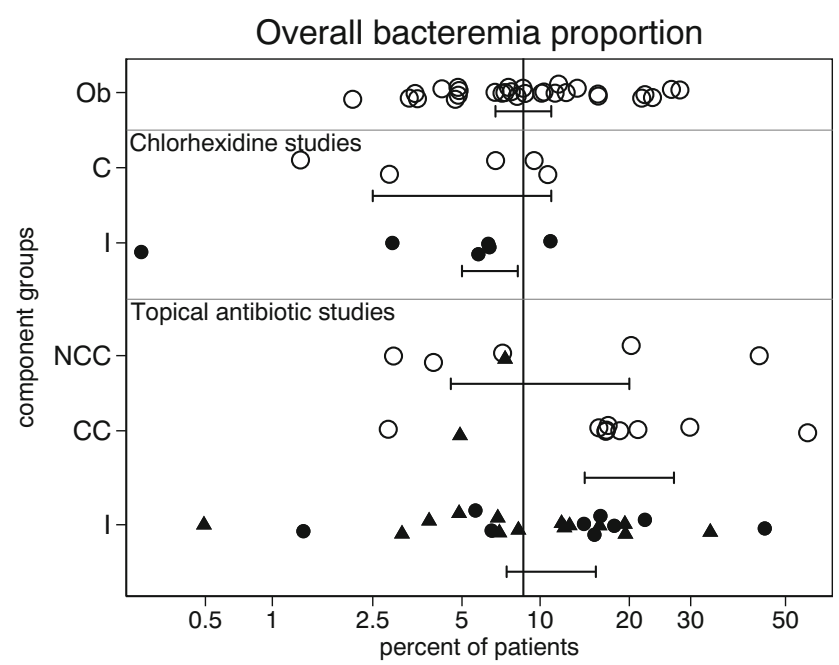

Fig. 2 Incidence of overall bacteremia versus benchmark. The overall bacteremia incidence for the control $(\mathrm{C}$, open symbols) and intervention (I, solid symbols) component groups from studies of either chlorhexidinebased or antibiotic-based methods versus the benchmark being the summary mean (central vertical line) derived from the observation studies (open circles). The 95\% confidence limits for mean incidence are shown for each stratum. Among component groups of antibiotic studies are control (CC and $\mathrm{NCC}$ ) and intervention groups that received PPAP (solid triangles) or intervention groups that received only TAP (solid circles). All other control groups received neither (open circles). Note that the $x$ axis is a logit scale. The plot is shown in more detail in Fig. S2

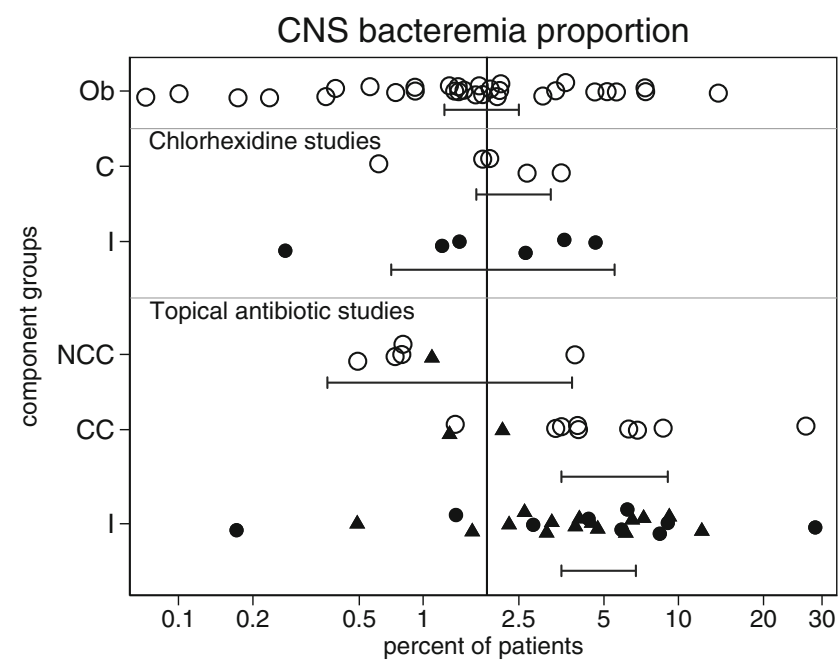

Fig. 3 Incidence of CNS bacteremia versus benchmark. The CNS bacteremia incidence for the component groups from studies of either chlorhexidine-based or antibiotic-based methods versus the benchmark being the summary mean (central vertical line) derived from the observation studies (open circles). Symbol coding is as in the Fig. 2 legend. Note that the $x$-axis is a logit scale and the continuity correction is used to enable a plot of those groups with a zero count. The plot is shown in more detail in Figs. S5 and S6

\section{Discussion}

The CNS bacteremia benchmark derived here is $1.8 \%$ (Table 1). The incidence is higher among studies applying less stringent diagnostic criteria for CNS bacteremia and among both intervention and $\mathrm{CC}$ control (but not NCC control) groups of antibiotic-based decontamination studies. The incidence in studies of ICU populations with a short mean length of stay, which were excluded from the analysis, is usually lower (Table S4).

Being an uncommon and variably defined end-point, the study of CNS bacteremia and the identification of risk factors is challenging. This study attempts to provide a novel, group level perspective on the impact of various exposures on CNS bacteremia incidences reported in the ICU literature. The exposures of interest here, decontamination interventions and diagnostic criteria for CNS bacteremia, can be regarded as group allocated. That is, they are received, or not, by all members of the group. Moreover, the control groups of the SDD/ SOD studies may or may not be concurrent to an ICU context in which topical antibiotic is in use. This arrangement enables testing the concurrency of control patients to TAP use in the ICU environment might serve as a contextual exposure. In this regard, the studies here can be regarded as a natural experiment resembling a cluster-randomized trial [18].

Natural experiments are those in which a differential population exposure, often unplanned, enables an analysis of consequences, often unforeseen, which otherwise might be difficult to study. For harmful outcomes, the intervention might otherwise be unethical to undertake as an intentional study. 
Table 2 Meta-regression models ${ }^{\mathrm{a}, \mathrm{b}, \mathrm{c}}$

\begin{tabular}{|c|c|c|c|c|c|c|}
\hline & & Overall bacteremic & & & CNS bacteremia & \\
\hline Factor & Coef $^{d}$ & $95 \% \mathrm{CI}$ & $p$ & Coef. $^{\mathrm{d}}$ & $95 \% \mathrm{CI}$ & $p$ \\
\hline $\begin{array}{l}\text { Groups from observational } \\
\text { studies (reference group) }\end{array}$ & -2.3 & $-3 \cdot 0$ to -1.6 & 0.001 & -4.1 & $-5 \cdot 0$ to -3.1 & 0.001 \\
\hline \multicolumn{7}{|l|}{ Chlorhexidine studies } \\
\hline - Control groups & -0.55 & -1.5 to +0.40 & 0.25 & -0.04 & -1.2 to +1.09 & 0.94 \\
\hline - Intervention groups & -0.46 & -1.35 to +0.44 & 0.31 & -0.03 & -1.05 to +0.98 & 0.95 \\
\hline \multicolumn{7}{|l|}{ Antibiotic studies } \\
\hline - NCC control groups & -0.09 & -0.68 to +0.86 & 0.82 & -0.46 & -1.49 to +0.57 & 0.37 \\
\hline - $\mathrm{CC}$ control groups & +0.80 & +0.15 to +1.46 & 0.017 & +1.15 & +0.41 to +1.9 & 0.003 \\
\hline - Intervention groups & +0.22 & -0.27 to +0.70 & 0.37 & +0.88 & +0.33 to +1.42 & 0.002 \\
\hline MV for $>48 \mathrm{~h}$ for $>90 \%^{\mathrm{e}}$ & +0.22 & -0.27 to +0.70 & 0.37 & +0.25 & -0.27 to +0.78 & 0.34 \\
\hline Stringent diagnostic criteria ${ }^{f}$ & & & & -0.53 & -0.16 to -0.42 & 0.034 \\
\hline Year of publication ${ }^{\mathrm{g}}$ & -0.09 & -0.34 to +0.15 & 0.43 & +0.13 & -0.18 to +0.37 & 0.38 \\
\hline
\end{tabular}

${ }^{a}$ These models are derived using random effects methods. The findings from a generalized estimating equation model were similar (data not shown)

${ }^{\mathrm{b}} I C U$ intensive care unit, $L O S$ length of ICU stay, $N C C$ non-concurrent control, $C C$ concurrent control, $M V$ mechanical ventilation

${ }^{\mathrm{c}}$ Repeating the meta-regression model to incorporate two senstivivity tests as mentioned in the methods gave similar findings

${ }^{\mathrm{d}}$ Interpretation. For each model, the reference group is the observational study (benchmark) groups and this coefficient equals the difference in logits from 0 (a logit equal to 0 equates to a proportion of $50 \%$; a logit equal to -2.2 equates to a proportion of $10 \%$; a logit equal to -4.6 equates to a proportion of $1 \%$ ) and the other coefficients represent the difference in logits for groups positive for that factor versus the reference group

${ }^{\mathrm{e}}$ The coefficient representing the increment for groups for which $>90 \%$ of patients received mechanical ventilation for $>48 \mathrm{~h}$

${ }^{\mathrm{f}}$ The coefficient representing the increment for groups with stringent diagnostic criteria for CNS bacteremia (documented in at least two blood cultures)

${ }^{\mathrm{g}}$ Year of study publication with the coefficient representing the increment for each decade post 1980

Examples of natural experiments with population-wide exposures to antibiotics are rare [19].

This natural experiment enables a study of the impact of various exposures on CNS bacteremia in this patient population. Most studies here were undertaken among ICU patients receiving MV with the ventilator-associated pneumonia incidence being the foreseen end-point. Only in the last decade has the overall bacteremia incidence among the broader ICU patient population including those without MV emerged as a primary study endpoint among these decontamination studies. The CNS bacteremia incidence was the primary end-point for none of the studies here.

This natural experiment recapitulates previous analyses of these studies of decontamination interventions that have found multiple paradoxical observations [20-22]. Specifically, several bacteremia and other end-points are higher among concurrent control (CC) groups versus non-concurrent control (NCC) groups of SDD/SOD studies presumably in association with contextual (indirect) exposure to the TAP use within the ICU.

\section{Limitations}

There are five key limitations to this analysis. Firstly, there are multiple decontamination agents, regimens, and strategies and not all studies were sourced from systematic reviews. Second, the studies have been published over three decades with consequent heterogeneity in the populations and study designs. Several had novel study designs with one or more intervention groups and either one or no control group. Hence, given these two limitations, the summary effect sizes derived here as displayed in Fig. S1 are indicative and intended for internal reference only. Of note, however, is that the summary effect estimates of these decontamination interventions on overall bacteremia here is similar to that in recent respective systematic reviews [14, 15].

Thirdly, the analysis is inherently observational and there is no ability to adjust for differences between groups in underlying patient-level risk. By benchmarking the incidences of CNS bacteremias in the component groups of studies of decontamination studies versus studies of comparable patient groups in the literature, this analysis takes a group level rather than patient-level approach to examine the question of risk factors. Of note, the strength of an exposure as a risk factor will differ for a subjectspecific estimate, as in a case control study, versus a populationaveraged estimate, as in the analysis here. The latter estimate could be expected to be weaker.

Fourthly, the allocation to decontamination in several studies was either cluster-randomized or non-random, more so for 
NCC studies. Moreover, exposure to other potential risk factors for CNS bacteremia would likely have occurred among the patients in all groups and remains unmeasured. For example, antibiotic therapies given as therapeutic interventions may be a compounding factor towards the acquisition of CNS colonization and bacteremia. Moreover, any association with prolonged mechanical ventilation prevalence may represent mechanical ventilation serving as a surrogate marker for other invasive devices, such as prevalence of central venous line use. These unspecified and unmeasured exposures would likely bias the findings here towards the null.

Finally, are the findings here robust to possible publication bias and undiscovered data? There are at least another 17,000 control and intervention group patients among two large studies of SDD/SOD in the literature for which CNS bacteremia data was not available [23, 24]. Assuming incidences equivalent to $1.3 \%$ (i.e., stringent criteria) would give an additional $221 \mathrm{CNS}$ bacteremias. Tallying these hypothetical with the known CNS bacteremias $(n=555)$ among the 10,986 intervention and CC control group patients of the SDD/SOD studies as noted here (Table S3) would give 776 CNS bacteremias among a hypothetical total of 27,986 $(10,986+17,000)$ patients for an overall hypothetical incidence of $2.8 \%$, which does not quench the excess.

The findings here are generally consistent with findings reported elsewhere. A non-randomized study of SDD among hematology patients found substantial differences in CNS bacteremia incidence defined using stringent criteria among the 313 bacteremia isolates over 6 years [25]. The CNS bacteremia incidence rate was approximately sixfold higher in hematology wards using versus not using SDD. While the time trend here was not significant, other studies have found an increasing incidence of CNS bacteremia over this time period [1-3]. The findings with overall bacteremia here resemble the findings for overall bacteremia among larger panels of $>100$ studies [16, 17]. Moreover, the findings with $S$. aureus bacteremia here resemble the findings for $S$. aureus VAP among a larger panel of 198 studies [22].

The likely explanation for the high incidence of CNS bacteremia within the concurrent control and intervention groups of SDD/SOD studies is through an altered ICU ecology as an indirect effect of antibiotic use. Moreover, there is a rebound colonization following withdrawal of SDD/SOD and whether CNS forms part of this rebound remains unclear [26]. The effect of ICU contextual, or "whole-of-ICU," exposure can be difficult to estimate except as a natural experiment [27].

\section{Conclusion}

Decontamination studies among adult ICU patients provide a natural experiment of group level risk factors for CNS bacteremia. Surprisingly, CNS bacteremia is increased in both intervention and $\mathrm{CC}$ but not NCC groups of the antibiotic but not chlorhexidine decontamination studies versus groups from studies without an intervention in the literature. This implicates an indirect effect of antibiotic use on CNS bacteremia within the ICU context. The size of this effect appears to equal or exceed the size of several other key determinants.

Funding information This research has been supported by the Australian Government Department of Health and Ageing through the Rural Clinical Training and Support (RCTS) program.

\section{Compliance with ethical standards}

Conflict of interest The author declares that he has no conflict of interest.

\section{References}

1. Thylefors JD, Harbarth S, Pittet D (1998) Increasing bacteremia due to coagulase-negative staphylococci: fiction or reality? Infect Control Hosp Epidemiol 19(8):581-589

2. Hugonnet S, Harbarth S, Ferriere K, Ricou B, Suter P, Pittet D (2003) Bacteremic sepsis in intensive care: temporal trends in incidence, organ dysfunction, and prognosis. Crit Care Med 31(2):390 394

3. Pittet D, Wenzel RP (1995) Nosocomial bloodstream infections: secular trends in rates, mortality, and contribution to total hospital deaths. Arch Intern Med 155(11):1177-1184

4. Rahkonen M, Luttinen S, Koskela M, Hautala T (2012) True bacteremias caused by coagulase negative Staphylococcus are difficult to distinguish from blood culture contaminants. Eur J Clin Microbiol Infect Dis 31(10):2639-2644

5. Widerström M, Wiström J, Sjöstedt A, Monsen T (2012) Coagulase-negative staphylococci: update on the molecular epidemiology and clinical presentation, with a focus on Staphylococcus epidermidis and Staphylococcus saprophyticus. Eur J Clin Microbiol Infect Dis 31(1):7-20

6. Favre B, Hugonnet S, Correa S, Sax H, Rohner P, Pittet D (2005) Nosocomial bacteremia: clinical significance of a single blood culture positive for coagulase-negative staphylococci. Infect Control Hosp Epidemiol 26:697-702

7. Park SY, Kwon KH, Chung JW, Huh HJ, Chae SL (2015) Coagulase-negative staphylococcal bacteremia: risk factors for mortality and impact of initial appropriate antimicrobial therapy on outcome. Eur J Clin Microbiol Infect Dis 34(7):1395-1401

8. Martin MA, Pfaller MA, Wenzel RP (1989) Coagulase-negative staphylococcal bacteremia: mortality and hospital stay. Ann Intern Med 110(1):9-16

9. Horan TC, Andrus M, Dudeck MA (2008) CDC/NHSN surveillance definition of health care-associated infection and criteria for specific types of infections in the acute care setting. Am J Infect Control 36(5):309-332

10. Van der Bij AK, Frentz D, Bonten MJ, Stuart JC, van Hees BC, Vijfhuizen J, Wintermans RG, der Kuil WA, Alblas J, van der Bij AK, ISIS-AR Study Group (2015) Gram-positive cocci in Dutch ICUs with and without selective decontamination of the oropharyngeal and digestive tract: a retrospective database analysis. J Antimicrob Chemother 71:816-820

11. Saunders GL, Hammond JM, Potgieter PD, Plumb HA, Forder AA (1994) Microbiological surveillance during selective 
decontamination of the digestive tract (SDD). J Antimicrob Chemother 34:529-544

12. Hurley JC (1995) Prophylaxis with enteral antibiotics in ventilated patients: selective decontamination or selective cross-infection? Antimicrob Agents Chemother 39:941-947

13. Klompas M, Speck K, Howell MD, Greene LR, Berenholtz SM (2014) Reappraisal of routine oral care with chlorhexidine gluconate for patients receiving mechanical ventilation: systematic review and meta-analysis. JAMA Intern Med 174:751-761

14. Silvestri L, Van Saene HK, Milanese M, Gregori D, Gullo A (2007) Selective decontamination of the digestive tract reduces bacterial bloodstream infection and mortality in critically ill patients. Systematic review of randomized, controlled trials. J Hosp Infect 65:187-203

15. Silvestri L, Weir WI, Gregori D, Taylor N, Zandstra DF, van Saene JJ, van Saene HK (2017) Impact of oral chlorhexidine on bloodstream infection in critically ill patients: systematic review and meta-analysis of randomized controlled trials. J Cardiothorac Vasc Anesth 31(6):2236-2244

16. Hurley JC (2014) Topical antibiotics as a major contextual hazard toward bacteremia within selective digestive decontamination studies: a meta-analysis. BMC Infect Dis 14:1

17. Hurley JC (2019) Studies of Selective digestive decontamination as a natural experiment to evaluate topical antibiotic prophylaxis and cephalosporin use as population-level risk factors for enterococcal bacteremia among ICU patients J Antimicrob Chemother (in press)

18. Hurley JC (2019) How the cluster randomized trial 'works'. Clin Infect Dis (in press)

19. Vandenbroucke-Grauls CM (2014) Antimicrobial resistance in the Netherlands: a natural experiment? Frontiers in Pub Health 2:5

20. Hurley JC (2011) Paradoxical ventilator associated pneumonia incidences among selective digestive decontamination studies versus other studies of mechanically ventilated patients. Benchmarking systematic review data. Crit Care 15:R7
21. Hurley JC (2018) Unusually high incidences of Pseudomonas bacteremias within topical polymyxin based decolonization studies of mechanically ventilated patients: benchmarking the literature. Open Forum Infect Dis 5:ofy 256

22. Hurley JC (2018) Unusually high incidences of Staphylococcus aureus infection within studies of ventilator associated pneumonia prevention using topical antibiotics: benchmarking the evidence base. Microorganisms 6:2

23. De Smet AM, Kluytmans JA, Cooper BS, Mascini EM, Benus RF, Van der Werf TS, Van der Hoeven JG, Pickkers P, Bogaers-Hofman D, Van Der Meer NJ, Bernards AT (2009) Decontamination of the digestive tract and oropharynx in ICU patients. N Engl J Med 360: 20-31

24. Oostdijk EA, Kesecioglu J, Schultz MJ, Visser CE, De Jonge E, van Essen EH, Bernards AT, Purmer I, Brimicombe R, Bergmans D, van Tiel F (2017) Notice of retraction and replacement: Effects of decontamination of the oropharynx and intestinal tract on antibiotic resistance in ICUs: a randomized clinical trial. JAMA 312:14291437

25. Daxboeck F, Rabitsch W, Blacky A, Stadler M, Kyrle PA, Hirschl AM, Koller W (2004) Influence of selective bowel decontamination on the organisms recovered during bacteremia in neutropenic patients. Infect Control Hosp Epidemiol 25:685-689

26. Tetteroo GW, Wagenvoort JH, Bruining HA (1994) Bacteriology of selective decontamination: efficacy and rebound colonization. J Antimicrob Chemother 34:139-148

27. Hurley JC (2019) Is selective decontamination (SDD/SOD) safe in the ICU context? J Antimicrob Chemother 74:1167-1172

Publisher's note Springer Nature remains neutral with regard to jurisdictional claims in published maps and institutional affiliations. 